\title{
A rare case of primary penile leiomyosarcoma: A case report
}

\author{
Anjali Sharma ${ }^{1}$, Sanjeev Patni ${ }^{2}$, Arpita Mathur ${ }^{3}$, Syeda Firdos Jamil ${ }^{3}$, Simran Gilhotra ${ }^{4}$, Hitesha Bhandari ${ }^{4}$ \\ From ${ }^{1}$ Senior Consultant, Director and Head of Department, ${ }^{3}$ Senior Resident, ${ }^{4}$ DNB Resident, Department of Pathology, Bhagwan Mahaveer Cancer \\ Hospital and Research Centre, Jaipur, Rajasthan, India, ${ }^{2}$ Senior Consultant, Director and Head of Department, Department of Surgical Oncology, \\ Bhagwan Mahaveer Cancer Hospital and Research Centre, Jaipur, Rajasthan, India
}

\begin{abstract}
Primary penile leiomyosarcoma is a rare tumor and very little data are available in the medical literature related to its behavior and potential outcome. A good surgical approach is essential for better disease-free survival. In our case study, a 50-year-old male presented with a 3-month history of penile swelling. The patient was treated with conservative surgery and immunohistochemistry played a key role in its diagnosis. No adjuvant therapy was given and the patient presented with a good surgical outcome after 6 months of follow-up. We present here a case report of this rare entity along with a short review of the literature including all the differential diagnoses of penile spindle cell lesions.
\end{abstract}

Key words: Immunohistochemistry, Penile leiomyosarcoma, Spindle cell sarcoma

$\mathrm{P}$ rimary soft-tissue sarcomas (STSs) of the genitourinary (GU) tract account for only $2.1 \%$ of STSs and $1-2 \%$ of all malignant GU tumors [1]. Penile sarcomas are rare and represent $<5 \%$ of penile malignancies (most of them are of vascular origin) [2]. Kaposi's sarcoma, epithelioid hemangioendothelioma, and angiosarcoma being more common and less frequently than rhabdomyosarcoma and leiomyosarcoma, of which leiomyosarcoma represents approximately 5-6\% of penile sarcomas [3]. The known predisposing factors are phimosis, cigarette smoking, and human papillomavirus infection.

Here, we report a rare case of penile leiomyosarcoma in which a patient presented with a lesion over glans. Furthermore, this case highlights the significance of immunohistochemistry (IHC) in the diagnosis of this rare entity if correlated carefully with typical histological features.

\section{CASE REPORT}

A 50-year-old male patient presented with a complaint of painful penile swelling associated with watery discharge. The pain was of severe intensity. The growth was present for 3 months but increased in size and became painful in the past few weeks.

General examination revealed no abnormality and all the vital signs were normal. On examination of the bilateral inguinal

\section{Access this article online}

Received - 07 April 2021

Initial Review - 22 April 2021

Accepted - 24 May 2021

DOI: $10.32677 /$ IJCR.2021.v07.i06.002 region, there was no significant lymphadenopathy. There were no associated comorbidities. On local physical examination, the growth was approximately $6 \mathrm{~cm}$ in size and ulceroproliferative over the anterior surface of the distal half of the shaft of the penis, just extending onto the proximal part of the glans.

All routine blood investigations were normal except the patient being reactive for human immunodeficiency virus antigen. Ultrasonography of the inguinal region revealed mildly enlarged lymph nodes that were reactive in nature. A small biopsy from the penile mass suggested a high-grade spindle cell tumor.

The mass was excised through the partial amputation of the penis. No lymphadenectomy was done due to $\mathrm{N}-0$ status. On gross examination, a nodular proliferative growth was identified measuring $6.1 \mathrm{~cm} \times 5.5 \mathrm{~cm} \times 2.4 \mathrm{~cm}$. The tumor was involving the glans, coronal sulcus, and the adjacent skin. The cut surface was poorly circumscribed, firm, and gray-white in color. The ulcerated area was infiltrating into the corpora spongiosa and was reaching up to the urethra (Fig. 1).

On microscopy, a highly cellular spindle cell neoplasm was identified comprising spindloid to ovoid cells with moderate-to-severe nuclear pleomorphism. The tumor cells were arranged in fascicles forming a storiform pattern at places. Occasional myxoid areas were also seen. Mitotic rate was 11-13/10 high-power fields. Overall morphology favored high-grade malignant spindle cell neoplasm.

The tumor was involving the glans invading the corpora spongiosa and the adjacent corpora cavernosa. The tumor was

Correspondence to: Dr. Arpita Mathur, Department of Pathology, Bhagwan Mahaveer Cancer Hospital and Research Centre, Jaipur, Rajasthan, India. E-mail: arpitamathur30@gmail.com

(C) 2021 Creative Commons Attribution-NonCommercial 4.0 International License (CC BY-NC-ND 4.0). 
reaching up to the subepithelial tissue of the overlying skin and producing nodule. All the surgical margins including skin cut end, soft-tissue cut end, shaft cut end, and urethral cut end were free from the tumor involvement.

IHC was positive for smooth muscle antibody, caldesmon, cluster of differentiation (CD) 163, and negative for S100, and TLE1 (Fig. 2). Overall histopathological appearance and IHC profile suggested the diagnosis of leiomyosarcoma. Urethral mucosa and resection margins were free, the closest being $0.5 \mathrm{~cm}$. No adjuvant therapy was given. After 6 months of follow-up, the patient is disease free with no signs of recurrence.

\section{DISCUSSION}

This exceptionally rare entity was first highlighted by Levi in 1930 [4]. Till now, very few cases of primary penile leiomyosarcoma have been reported in the medical literature [5]. Penile leiomyosarcoma can occur at any age, but the highest incidence is in the fourth and fifth decades. Similar to the leiomyosarcoma at other common sites, these are well-circumscribed with a firm to rubbery consistency.

Depending on the invasion of the tunica albuginea, Pratt and Ross classified penile leiomyosarcomas as superficial and deep types [6]. Lesions arising from the dartos muscle layer, the piloerector complex, and the muscular walls of superficial vessels situated outside the albuginea are the superficial types while the lesions arising from the proximal portions of the corpora cavernosa or corpus spongiosum are deep types. Superficial types present as a small tumor in the distal shaft or the penile prepuce and often occur in middle-aged men. They grow slowly and have more tendency to recur locally although

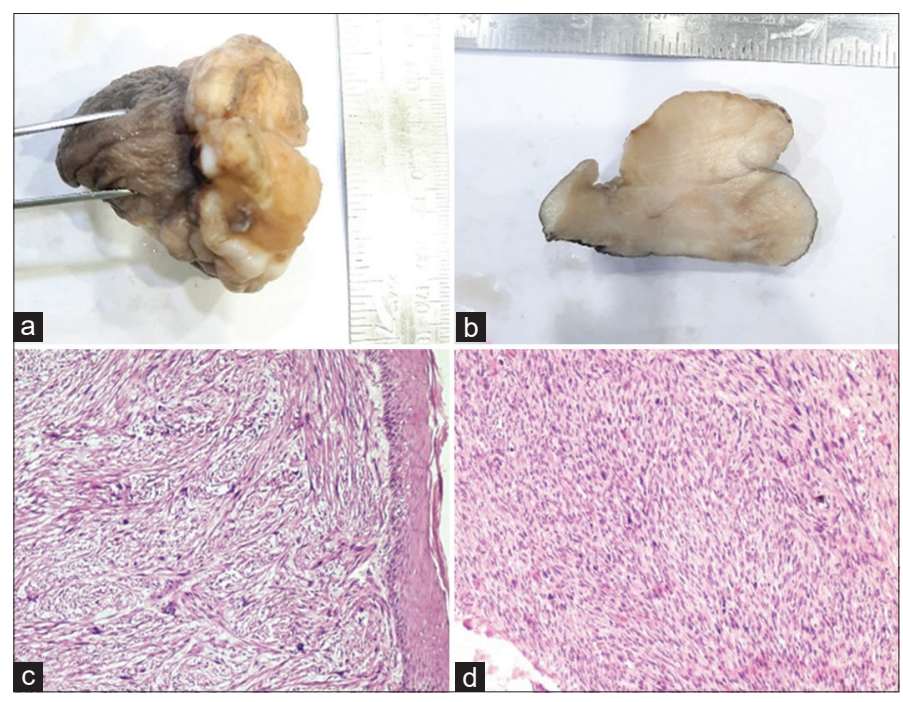

Figure 1: Photomicrograph showing gross specimen and microscopic appearance. (a) Gross specimen of partial penectomy revealing a nodular proliferative growth involving glans, coronal sulcus, and adjacent skin. (b) Cut surface showing poorly circumscribed, firm, and gray-white lesion infiltrating into corpora spongiosa and reaching up to urethra. (c and d) Scanner view $(\times 10)$ of $H$ and $E$ stained slide revealing a high-grade malignant spindle cell neoplasm arranged in fascicles and forming storiform pattern distant metastasis is uncommon. On the other hand, deep types are poorly circumscribed, firm, non-tender masses that infiltrate surrounding tissues and sometimes result in urinary obstruction. They occur at a relatively later age, show a greater propensity to metastasize, and have a poorer prognosis [7]. Histologically, both the types are identical.

Microscopically, malignant spindle cells are seen some with smooth muscle differentiation and arranged in interlacing fascicles. On electron microscopy, myofibrils, dense bodies, and abundant pinocytic vesicles are noted with a continuous basal lamina [8]. Mitotic rate and degree of differentiation are the most important factors in predicting the tumor propensity to infiltrate the adjacent structures or to metastasize.

IHC findings play a crucial role in the differential diagnosis of penile sarcomas and arriving at a definitive diagnosis. The most common differential diagnosis includes Kaposi sarcoma characterized by strong immunoreactivity for CD31 and CD34 and negative reactivity for desmin. Malignant fibrous histiocytoma rarely arises in the penis. It exhibits a prominent storiform-pleomorphic pattern and shows negative reactivity for actin and desmin. Sarcomatoid carcinoma of the penis exhibits cellular atypia of the surface epithelium and immunoreactivity for keratin.

Complete surgical resection is an optimal approach for the treatment of this rare malignancy resulting in better survival. Recurrences in superficial tumors are operable because they often remain localized for a long time before giving rise to metastases while deep-seated tumors require amputation either partial or radical with perineal urethrostomy [7]. Regional lymph node dissection is typically not indicated in the absence of clinicoradiologically negative lymph nodes as nodal metastasis is rare. As suggested by Fetsch et al. in a review of 14 cases of penile leiomyosarcoma, local recurrence is a frequent

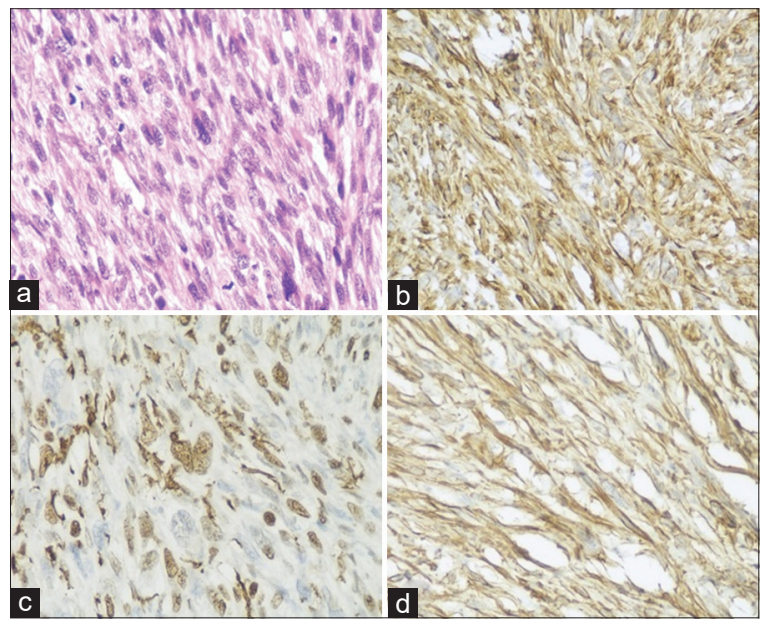

Figure 2: Photomicrographs showing spindle cells and their phenotypical expression. (a) High-power view $(\times 40)$ depicting malignant spindle cells with moderate-to-severe nuclear pleomorphism. High mitotic rate also seen. (b) Immunohistochemistry (IHC) - strong and diffuse cytoplasmic immunoreactivity for caldesmon. (c) IHC CD 163 - positive in tumor cells and (d) IHC strong and diffuse cytoplasmic immunoreactivity for Smooth Muscle Actin (SMA). 
phenomenon and it becomes more undifferentiated with each recurrence [7]. They also concluded that superficial and deep lesions have similar recurrence rates, $23 \%$ and $29 \%$, respectively, although the metastatic potential is higher in deep-seated lesions $(50 \%)$.

The most frequent sites of distant metastases are the lungs, liver, and brain. Few case reports suggest that adjuvant radiation and chemotherapy are not required in the treatment of primary lesion neither in reducing locoregional recurrences nor in increasing survival rates [9]. While, there are some studies that show local recurrence in deep lesions in absence of adjuvant therapy [10]. At present, radiotherapy is used for palliation, with chemotherapy is reserved for cases of disseminated disease. The metastatic potential for smaller lesions $(<5 \mathrm{~cm})$ is $29 \%$ while for larger lesions (more than $5 \mathrm{~cm}$ ) is $50 \%$. The most important parameters deciding the prognosis are the size and the site of the tumor. Large tumors and those located at the root of the penis have a poor prognosis.

\section{CONCLUSION}

There are no standard guidelines regarding the treatment protocol of this rare tumor, however, upfront complete surgical excision is advised. We are presenting this case of spindle cell sarcoma of the penis, as it is an exceedingly rare malignancy. In the Indian literature, there is little information available on the clinical and pathological aspects of this entity.

\section{REFERENCES}

1. Stojadinovic A, Leung DH, Allen P, Lewis JJ, Jaques DP, Brennan MF. Primary adult soft tissue sarcoma: Time-dependent influence of prognostic variables. J Clin Oncol 2002;20:4344-52.

2. Lucia MS, Miller GJ. Histopathology of the malignant lesions of the Penis. Urol Clin North Am 1992;19:227-46.

3. Dominici A, Rose AD, Stomaci N, Pugliese L, Posti A, Nesi G. A rare case of leiomyosarcoma of the penis with a reappraisal of the literature. Int J Urol 2004;11:440-4.

4. Levi I. On a case of primary fibrosarcoma of the skin of the penis: Clinical and histological study. G Ital Dermatol Venereol 1930;71:1559-74.

5. Dhull AK, Kaushal V, Dalal S, Singh S. The inside mystery of penile leiomyosarcoma. Indian J Cancer 2015;52:241-2.

6. Pratt RM, Ross RT. Leiomyosarcoma of the penis. A report of a case. Br J Surg 1969;56:870-2.

7. Fetsch J, Davis CJ, Miettinen M, Sesterhenn I. Leiomyosarcoma of the Penis: A clinicopathologic study of 14 cases with review of the literature and discussion of the differential diagnosis. Am J Surg Pathol 2004;28:115-25.

8. Khobragade KH, Tamhankar AS, Bakshi GK, Tongaonkar HB, Menon S. Leiomyosarcoma of penis. Indian J Cancer 2015;52:374-5.

9. Gonzalez EJ, Jiménez JL, Pineda MP, Morán AM, Fernández JC. Leiomyosarcoma of the penis, an exceptional entity. Urol Case Rep 2015;3:63-4.

10. Thomas K, Spiess P, Caracciolo JT. A report of two deep-seated noncutaneous penile tumors: More than meets the eye. Radiol Case Rep 2017;12:726-30.

Funding: None; Conflicts of Interest: None Stated.

How to cite this article: Sharma A, Patni S, Mathur A, Jamil SF, Gilhotra S, Bhandari H. Arare case of primary penile leiomyosarcoma: A case report. Indian J Child Reports. 2021;7(6):226-228. 